\title{
Temporal Interaction between an Artificial Orchestra Conductor and Human Musicians
}

\author{
Dennis Reidsma \\ Anton Nijholt \\ Pieter Bos \\ University of Twente, Human Media Interaction \\ Faculty EEMCS, University of Twente, Enschede, the Netherlands.
}

\section{Introduction}

One interest of our research group is in (1) developing Ambient Entertainment technologies and applications that (2) interact in a coordinated way with human partners using (3) a multitude of different sensors observing many characteristics of the partner and using (4) many different channels of expression (such as sound, visuals, speech and embodiment with gestures and facial expressions). The Virtual Conductor project $[1,2]$ concerns the development of the first properly interactive virtual orchestra conductor that can conduct a piece of music through interaction with musicians, leading and following them while they are playing. Its observations consist of different manners of musical processing of the incoming signal from a microphone. The forms of its expressions are defined by the possible conducting patterns and by the timing, speed, amplitude and smoothness with which those patterns are expressed. The interaction is focused on the tempo of the musicians and includes a correction module that interactively corrects the tempo when the musicians are playing too slow or too fast.

We describe our motivation for developing such a system; related work in the areas of ambient entertainment and coordinated timing, automatic music processing, virtual humans and conducting; the design, implementation and evaluation of the Virtual Conductor; and, finally, contains a discussion of the resulting system, general applicability of the ideas and technology developed in this project, and expected developments in the (ongoing) Virtual Conductor project.

\section{Motivation}

More and more, music is becoming a theme in computer based entertainment. Games in which interaction with or through music plays a central role are on the rise (see, for example, games such as Guitar Hero, Dance Dance Revolution, Donkey Konga and many, many more). However, for many of those games the interaction through music is mostly one-way: the player must follow a rhythm or riff presented by the computer to achieve a set goal. When a group of people make music, interaction is inherently simultaneous and two-way. Both partners in a musical cooperation are alert to what the other is doing and adapt their own performance to mesh.

In the Virtual Conductor project presented in this paper a major element is the mutual interaction between system and musician with respect to tempo and timing. Some ideas concerning temporal coordination in interaction have been worked out preliminarily in [3], in relation to topics such as synchrony. Here we just note the strong positive relation found in literature between synchrony and positive affect, or between synchrony and a positive evaluation of the interaction, in human-human interaction, but also in human-computer interaction. Given the literature it seems a reasonable assumption that implementation of modules for synchrony can add to the enjoyment and engagement of users of computational entertainment applications. The Virtual Conductor can be seen as one of the first ambient entertainment applications that takes a step in the direction of harnessing interactional synchrony for improving the enjoyment and engagement of the user.

A Virtual Conductor system can be used in several ways. An edutainment application of such technology could be in teaching student conductors. As a reflective tool, the system could show good examples as 
well as examples of typical conducting mistakes or allow the student conductor to visualise different ways of conducting a passage to see what it looks like. In combination with the complement of this artificial conductor, namely an artificial orchestra such as the one on display in the Vienna House of Music, a system could be envisioned that detects the student's mistakes and graphically shows them to the student in combination with a better way of conducting. We can also envision this conductor developed further as a rehearsal conductor. The time in which a human conductor can work with a certain ensemble is often limited; if a Virtual Conductor could be used to rehearse the more technical aspects of a piece of music this would leave the human conductor more time to work on the creative and expressive musical aspects of a performance. Finally, a Virtual Conductor could also be made available through the internet to provide the casually interested layman with easy and engaging access to knowledge about, and some do-it-yourself experimentation with, conducting.

\section{Survey and Conclusions}

A Virtual Conductor has been researched, designed and implemented that can conduct human musicians in a live performance. The conductor can lead musicians through tempo, dynamics and meter changes. During test sessions the musicians reacted to the gestures of the conductor. It can interact with musicians in a basic way, correcting their tempo gracefully when they start playing faster or slower than is intended. Feedback from the musicians who participated in the tests shows that the musicians enjoy playing with the virtual conductor and can see many uses for it, for example as a rehearsal conductor when a human conductor is not available, or a conductor for playing along with when practicing at home.

Several audio algorithms have been implemented for tracking the music as played by the ensemble. Among those, the beat detector can track the tempo of musicians and the score follower can track where musicians are in a score, in real-time. The possibilities of these audio algorithms reach further than what is currently used for feedback in the Virtual Conductor and will be very useful for future extensions of the system.

While this article presents the results of the first stages of the Virtual Conductor project, the system is still being extended in several directions. We have been working on a detailed analysis of a corpus of performances and rehearsals that we recorded on video, in order to find out more about the intentions and signals used in conducting and about the structure of rehearsals. The repertoire of expressions that the Virtual Conductor can use is continuously being extended. We are working on implementing rehearsal modules that allow the Virtual Conductor to plan a structured series of rehearsal sessions, adapting later sessions in response to the progress achieved in the earlier sessions. Also, the planning and scheduling modules used to produce the conducting animations have been extensively redesigned in order to allow a much more flexible and responsive behavior generation that can handle several expressive channels at the same time. This will allow us to combine the basic beat patterns with an extensive repertoire of left hand gestures, facial expressions, gaze behavior and other non verbal expressions.

\section{References}

[1] D. Reidsma, A. Nijholt, A., and P. Bos. Temporal Interaction between an Artificial Orchestra Conductor and Human Musicians. ACM Journal on Computers and Entertainment, accepted for publication.

[2] M. ter Maat, R. Ebbers, D. Reidsma and A. Nijholt. Beyond the beat: modelling intentions in a virtual conductor. In: Proceedings of the 2nd International Conference on intelligent Technologies For interactive Entertainment (INTETAIN). ACM Digital Libraries, 1-10.

[3] A. Nijholt, D. Reidsma, H. van Welbergen, H.J.A. op den Akker, and Z.M. Ruttkay. Mutually Coordinated Anticipatory Multimodal Interaction. In: Nonverbal Features of Human-Human and HumanMachine Interaction, 29-31 October 2007, Patras, Greece. Lecture Notes in Computer Science 5042, Springer Verlag, Berlin, 2008, 73-93. 